\title{
Prognostic Factors Predicting Early Recovery of Pre-fracture Functional Mobility in Elderly Patients With Hip Fracture
}

\author{
Daegu Lee, MD, Jae Yong Jo, MD, Ji Sun Jung, MD, Sang Jun Kim, MD, PhD \\ Department of Physical and Rehabilitation Medicine, Samsung Medical Center, \\ Sungkyunkwan University School of Medicine, Seoul, Korea
}

Objective To investigate the prognostic factors predicting the recovery of pre-fracture functional mobility, we evaluated this by the use of ambulatory assistive devices in short-term follow-up.

Methods Five hundred and fifty-three elderly patients who had undergone hip fracture operations from January 2006 to June 2013 were enrolled in this retrospective study. Clinical characteristics and predicted factors affecting functional recovery, such as the delay of rehabilitation after the operation, were reviewed. The functional status of the gait was classified as either a bedridden state, wheelchair-bound state, walker gait, single cane gait, and selfgait without any ambulatory assistance device. When this functional grade in patients who recovered after the surgery was compared to before the surgery, this state was considered 'functional recovery'.

Results One hundred and ninety-two patients (34.7\%) showed recovery of preoperative mobility in the first month after their operation. Multiple logistic regression analysis identified that the following four factors were significantly associated with a deterioration of functional recovery: old age (odds ratio [OR], 0.95; 95\% confidence interval [CI], 0.92-0.97), delays in rehabilitation after operation (OR, 0.94; 95\% CI, 0.89-0.98), the presence of cognitive dysfunction (OR, 0.36; 95\% CI, 0.18-0.71), and trochanteric fracturing (OR, 0.58; 95\% CI, 0.36-0.94).

Conclusion We found that old age, cognitive dysfunction, trochanteric fracture type, and delay of rehabilitation were associated with the deterioration of functional recovery after a hip fracture operation in the short-term. Therefore, early rehabilitation was required to acquire functional recovery after a hip fracture operation in the short-term.

Keywords Hip fractures, Prognosis, Rehabilitation, Recovery of function

(c) This is an open-access article distributed under the terms of the Creative Commons Attribution Non-Commercial License (http://creativecommons.org/ licenses/by-nc/3.0) which permits unrestricted noncommercial use, distribution, and reproduction in any medium, provided the original work is properly cited. Copyright $\odot 2014$ by Korean Academy of Rehabilitation Medicine 


\section{INTRODUCTION}

Elderly people have a high risk of falling down because their proprioceptive function had decreased and their muscles were weakened [1]. According to a report from the Korean Geriatric Society in 2010, the incidence of falling down in people aged 65 years or over was $13 \%$, and this fall down was progressed into a hip fracture in $3.7 \%$ [2]. Recently, the incidence of hip fractures was rising worldwide due to the aging population [3]. Hip fractures in the elderly were catastrophic events that often lead to dramatic consequences, such as disability or death. It was also a major public health concern that placed a high economic burden on patients, families, and the medical system [4].

The ability to remain mobile was an essential aspect for quality of life and was critical for the preservation of independence in old age. Particularly in elderly patients with hip fracture, early mobilization was an important issue because ambulatory status was a predictive factor for one-year mortality after hip surgery [5]. In order to reduce functional decline and mortality after a hip fracture, efforts to identify predictable factors upon admission to the hospital needed to be increased. Some authors have suggested possible determinants or predictors of functional prognoses after hip fracture surgery. Various factors, such as male gender, low serum albumin (used as a measure of nutrition), impaired cognitive function, depression, and poor pre-fracture functional level, have been reported to be associated with an increased risk of functional deterioration after a hip fracture [6-9].

In previous reports, the parameters for the functional prognosis in these studies were from binary data such as mortality after surgery $[5,10]$, the gains of the surgery [11] to continuous data such as Functional Independence Measure [9,12], or cumulative ambulation score [13]. There have been no studies that have evaluated the functional mobility according to the use of ambulatory assistive device. According to the balance and weight-bearing capacity of a patient, he or she may need an ambulatory assistive device or may be able to walk independently. Previous reports have found that a single cane can support approximately one-quarter of a person's body weight [14]. Crutches and walkers can support approximately half of a person's body weight and improved a patient's balance by increasing the base of their support [14]
Using an assistive device, such as a walker, will prevent the elderly from slipping, but it had a disadvantage when stepping up or down or walking on uneven ground. In addition, using the upper extremities for the device when walking makes it difficult for a person to perform activities, such as opening or closing a door. Therefore, an elderly person who can walk without an ambulatory device felt more satisfied and comfortable with their quality of life than an elderly person who must use an ambulatory device when walking.

The aim of the present study was to identify prognostic factors to predict the recovery of pre-fracture functional mobility as evaluated by the use of ambulatory assistive device in patients with a hip fracture through short-term follow-ups. We also evaluated the patient characteristic, clinical outcomes, and factors that influence postoperative functional mobility in South Korea.

\section{MATERIALS AND METHODS}

\section{Subjects}

This study was a retrospective, longitudinal observational study. The data was collected from 599 patients, aged 65 years or more, who were admitted to the department of orthopedic surgery for a hip fracture and underwent hip joint surgery between January 2006 and June 2013. Patients with a fracture caused by secondary causes, such as bone metastatic cancer or Paget disease of the bone and those who had sustained a fracture due to a major trauma, were excluded. The functional status of 46 patients could not be evaluated because of a lack of medical records; therefore, 553 patients were included in the final analysis. After the surgery, all patients received physical therapy, which was performed by three experienced physical therapists using predetermined protocols during the admission period. Physical therapy consisted of physical modality to reduce postoperative pain, exercises to increase the range of motion and motor power of the lower legs, using a tilting table to adapt to standing gradually, and gait training. Gait training started from the walker gait and progressed to a single cane or selfgait according to the balance and weight-bearing capacities of patients under the guidance of two experienced physicians. Medication for pain control was prescribed after surgery, and its dose was adjusted according to pain status. Other medications for specific diseases that some 
patients suffered from were maintained during the admission period. Patients were discharged to their home, other local hospitals, or another department for further care or the management of medical problems. At one month after hip fracture surgery, the functional status of gait was reviewed through the medical records of physical therapists or physicians.

\section{Data collection}

The baseline information and status of patients was achieved by reviewing charts. All possible independent factors that could affect functional mobility after the hip fracture operation were evaluated. All medical records were used under the acceptance of patients, and all of these protocols were approved by the Institutional Review Board of our hospital (IRB No. 2011-11-049-002).

\section{Patient's factors}

Patient's factors including age, gender, depression, cognitive dysfunction, and pre-fracture functional level were obtained by retrospective chart review. Cognitive dysfunction was considered to be present when dementia or cognitive impairment had already been diagnosed before surgery, postoperative delirium appeared, or the score of the Mini-Mental Status Examination administered during the admission period was lower than 18 (demonstrating moderate-to-severe cognitive dysfunction, as suggested by a previous article [15]). Depression was considered present when depression was already diagnosed or medication was prescribed before the surgery.

\section{Surgery factor}

The time delay between fracture and operation (latency time), fracture type, surgery type, and the presence of postoperative complications were evaluated. Surgical procedures were categorized into closed reduction and internal fixation (CRIF), open reduction and internal fixation (ORIF), and hip replacement arthroplasty (HRA). Hip fractures were classified as femoral neck fracture or trochanteric fractures through the surgery records recorded by orthopedic surgeons who were well-experienced at hip fracture surgery. When the surgery was not restricted to the hip but also to other joints, it was considered as being multiple operations. Postoperative complications, such as pneumonia and delirium, were thoroughly investigated and recorded.

\section{Rehabilitation factor}

The time delay between operation and rehabilitation (latency time) and transference to the department of rehabilitation were evaluated.

\section{Outcome measures}

The use of ambulatory assistive devices was used to assess functional mobility level. The functional status of gait was classified as bedridden state (BR), wheelchairbound state (WC), hemi-walker or walker gait (WG), single cane gait (CG), or self-gait without an ambulatory assistance device (SG). These functional statuses were numerically graded, ranging from 1 to 5 , in descriptive order. When this functional grade in a patient was equal to, or exceeded one month after the surgery compared to before the surgery, this grade was considered 'early functional recovery'

\section{Statistical analysis}

Statistical analysis was performed with SPSS ver. 20.0 for Windows (IBM SPSS Inc., Armonk, NY, USA). To identify the patient's baseline characteristics and clinical outcomes, continuous variables, such as age, length of stay, time delay from fracture to operation (latency time), and time delay from operation to rehabilitation (latency time), were summarized as means and standard deviations. Categorical variables, such as gender, the presence of depression, the presence of cognitive dysfunction, fracture type, surgery type, and the presence of postoperative complications, were summarized as frequencies and percentages. The factors significantly associated with variables representing pre-fracture baseline conditions and factors associated with surgery were analyzed for identification. Correlation analysis between the functional mobility after hip surgery and independent variables was performed, and univariate logistic regression analysis was performed on each variable to determine the crude odds ratios of early functional recovery. The factors that showed significant differences $(\mathrm{p}<0.1)$ in univariate analysis were included as independent variables in the subsequent multivariate analysis. Multivariate analysis was performed by logistic regression using the abovementioned independent variables, and functional recovery as the dependent variable. Any p-values less than 0.05 were considered significant. 


\section{RESULTS}

In total, 553 patients were included in our study. The mean age of the subjects was 79.0 years, with a standard deviation of 7.0 years. Seventy-four percent of the subjects (414 patients) were female. Approximately 19\% (104 patients) of the fractures were femoral neck fractures, and $81 \%$ (449 patients) had sustained trochanteric fractures, with a corresponding ratio of trochanteric to femoral neck fracture patients of 4.31. The type of operation was CRIF ( $\mathrm{n}=276)$, ORIF $(\mathrm{n}=28)$, and HRA $(\mathrm{n}=249)$. The operation was done an average of $4.4 \pm 7.0$ days after the fracture, and the time from the operation to the physical therapy was $6.2 \pm 4.2$ days. The average duration of admission in our hospital was $23.0 \pm 16.6$ days. Medication was not included in this retrospective analysis because the type and dose of medication used in this study varied according to each patient's condition. The most commonly used medication was cetamadol, and the others were oxycodone, fentanyl patch, and non-steroidal antiinflammatory drugs, such as celecoxib, naproxen, and so on.

Before the surgery, the functional status of gait was SG in 318 patients, CG in 146 patients, WG in 59 patients, WC in 16 patients, and BR in 14 patients.

Twelve percent of the subjects (68 patients) had cognitive dysfunction, and 485 patients did not. Fifty-three patients had been diagnosed with dementia or cognitive impairment before their hip fracture, and 15 patients had postoperative delirium after the hip fracture operation. Major cognitive impairments were Alzheimer dementia $(n=25)$, vascular dementia $(n=14)$, dementia with Parkinson disease $(n=8)$, mild cognitive impairment $(n=5)$, and metabolic encephalopathy $(n=1)$. All patients with cognitive impairment were prescribed cognitive medication, such as rivastigmine, memantine, donepezil, and nicergoline, before their hip fracture. Among them, ten patients had their Mini-Mental Status Examination in their medical records and all scores were below 18 .

Major postoperative complications were infections in-

Table 1. Correlation of parameters and functional status of gait after hip surgery $(n=553)$

\begin{tabular}{lcc}
\hline \multicolumn{1}{c}{ Parameter } & Spearman $\rho$ & p-value* $^{*}$ \\
\hline Age & -0.163 & $<0.001$ \\
\hline Duration of admission & -0.129 & 0.002 \\
\hline $\begin{array}{l}\text { Day from fracture to operation } \\
\begin{array}{l}\text { Day from operation to } \\
\text { rehabilitation }\end{array}\end{array}$ & -0.180 & $<0.001$ \\
\hline $\begin{array}{l}\text { Pre-fracture mobility } \\
\text { Cognitive dysfunction }\end{array}$ & 0.192 & $<0.001$ \\
\hline Postoperative complication & -0.185 & $<0.001$ \\
\hline
\end{tabular}

*Spearman correlation test.

Table 2. Result of univariate analysis between patient characteristics and early functional recovery after hip fracture $(\mathrm{n}=553)$

\begin{tabular}{|c|c|c|c|c|}
\hline & \multirow{2}{*}{ No-recovery } & \multirow{2}{*}{ Recovery } & \multicolumn{2}{|c|}{ Univariate analysis } \\
\hline & & & Crude OR (95\% CI) & p-value* \\
\hline Age (yr) & $79.7 \pm 6.8$ & $77.7 \pm 7.3$ & $0.96(0.93-0.98)$ & 0.002 \\
\hline Day from operation to rehabilitation & $6.5 \pm 4.1$ & $5.5 \pm 4.4$ & $0.94(0.90-0.98)$ & 0.015 \\
\hline Cognitive dysfunction & & & & 0.041 \\
\hline Absence $(n=485)$ & $309(63.7)$ & $176(36.3)$ & 1 & \\
\hline Presence $(n=68)$ & $52(76.5)$ & $16(23.5)$ & $0.54(0.29-0.97)$ & \\
\hline Postoperative complication & & & & 0.105 \\
\hline Absence $(n=510)$ & $328(64.3)$ & $182(35.7)$ & 1 & \\
\hline Presence $(n=43)$ & $33(76.7)$ & $10(23.3)$ & $0.54(0.26-1.13)$ & \\
\hline Fracture type & & & & 0.072 \\
\hline Trochanter $(n=449)$ & $301(67.0)$ & $148(33.0)$ & 1 & \\
\hline $\operatorname{Neck}(n=104)$ & $60(57.7)$ & $44(42.3)$ & $1.49(0.96-2.30)$ & \\
\hline
\end{tabular}

Values are presented as mean \pm standard deviation or number (\%).

$\mathrm{OR}$, odds ratio; CI, confidence interval.

*Univariate binary logistic regression test. 
cluding pneumonia and urinary tract infection $(n=15)$, delirium ( $\mathrm{n}=15)$, skin problems including pressure sores and wound site oozing $(\mathrm{n}=4)$, azotemia $(\mathrm{n}=4)$, dyspnea including atelectasis $(\mathrm{n}=2)$, implant problems including $(\mathrm{n}=1)$, stroke $(\mathrm{n}=1)$, and ileus $(\mathrm{n}=1)$. The other 543 patients did not show any complications.

About one month after surgery, functional status was investigated through the medical records of the physician or physical therapists. Ninety-one patients could walk without any ambulatory device (SG), 108 patients walked with single cane (CG), and 186 patients walked with a rolling walker (WG). One hundred twenty-eight patients were in a wheelchair ambulation state, and 40 patients were bedridden. Six patients were dead because of the aggravation of underlying diseases. The one-month mortality rate for hip fracture was about $1 \%$.

Correlations between parameters and the functional status of gait after hip surgery are presented in Table 1. Age, duration of admission, days from fracture to operation, days from operation to rehabilitation, functional status of gait before hip surgery, cognitive dysfunction, and postoperative complications were correlated with the functional status of the gait after hip surgery.

The early recovery rate of functional mobility were compared with pre-fracture functional mobility was $34.7 \%$ (192 patients). Table 2 summarizes the association between the early recovery of functional mobility and patients' demographics and clinical characteristics. Univariate analysis revealed significant differences $(\mathrm{p}<0.1)$ for four variables-age, delay between operation and rehabilitation, cognitive dysfunction, and fracture type. No significant differences were observed for gender, depression, postoperative complication, or delays from fracture to operation. Postoperative complications $(\mathrm{p}=0.105)$ were included as independent variables in multivariate analy- sis. Therefore, five independent variables were included in the multivariate analysis.

When a dependent variable was set as a binary outcome by the presence of early functional recovery after operation, multiple logistic regression analysis identified four independent predictors-old age (odds ratio [OR], 0.95; 95\% confidence interval [CI], 0.92-0.97), delays in rehabilitation after operation (OR, 0.94; 95\% CI, 0.89-0.98), presence of cognitive dysfunction (OR, 0.36; 95\% CI, $0.18-0.71)$, and trochanteric fracture (OR, $0.58 ; 95 \% \mathrm{CI}$, 0.36-0.94). Factors found to be significantly associated with early postoperative functional recovery are summarized in Table 3.

Patients were divided into two groups according to their transfer to the rehabilitation department: 77 patients were transferred to the department of rehabilitation medicine for further physical therapy, and the other 476 patients were discharged to their home or another hospital. Table 4 summarizes the predictive factors of both patient groups. In particular, the proportion who recovered functional mobility early was similar between these two groups. The significant factors associated with transferal to the department of rehabilitation medicine are reported in Table 4.

Table 3. Risk factors predicting difficulties with functional recovery after hip fracture operation

\begin{tabular}{lcc}
\hline \multicolumn{1}{c}{ Predictive factor } & OR (95\% CI) & p-value* \\
\hline Age & $0.95(0.92-0.97)$ & $<0.001$ \\
$\begin{array}{l}\text { Day from operation to } \\
\text { rehabilitation }\end{array}$ & $0.94(0.89-0.98)$ & 0.015 \\
Cognitive dysfunction & $0.36(0.18-0.71)$ & $<0.001$ \\
\hline Trochanteric fracture & $0.58(0.36-0.94)$ & 0.028 \\
\hline
\end{tabular}

OR, odds ratio; $\mathrm{CI}$, confidence interval.

*Multivariate binary logistic regression analysis.

Table 4. Comparison of predictive factors between REH transfer group and no transfer group

\begin{tabular}{lccc}
\hline & Transfer to REH $(\mathbf{n}=\mathbf{7 7})$ & No transfer $(\mathbf{n = 4 7 6})$ & $\mathbf{p}$-value* \\
\hline Age & $79.9 \pm 7.9$ & $78.9 \pm 6.9$ & 0.453 \\
\hline Day from operation to rehabilitation & $6.5 \pm 4.5$ & $6.1 \pm 4.2$ & 0.883 \\
Cognitive dysfunction & $16(34.8)$ & $52(10.3)$ & $<0.001$ \\
\hline Trochanteric fracture & $34(73.9)$ & $415(81.9)$ & 0.187 \\
\hline Early recovery of functional mobility & $12(26.1)$ & $180(35.5)$ & 0.199 \\
\hline
\end{tabular}

Values are presented as mean \pm standard deviation or number (\%).

$\mathrm{REH}$, department of rehabilitation and physical medicine.

*Mann-Whitney U test for continuous variables and Pearson chi-square test for categorical variables. 


\section{DISCUSSION}

In the present study, we examined several prognostic factors for the early recovery of pre-fracture mobility in elderly patients after hip fracture. The value of the early prediction of functional outcomes after a hip fracture was important. It may assist in coordinating patients' and caregivers' expectations, and planning advanced care [16]. In addition, 'Inability to Walk at Hospital Discharge' was an independent predictor of mortality after hip surgery [5].

We assessed the patients' functional mobility through their use of ambulatory assistive devices. Walking with an ambulatory assistive device affected walking speed and distance, which were important contributors to the ability to perform daily activities and participate in a community [17]. Although ambulatory assistive devices have several advantages, the long-lasting use of inappropriate ambulatory assistive devices can have a negative impact on the patients $[17,18]$. Finding the corrective factors to predict functional mobility using an ambulatory assistive device could be helpful to increase the ambulatory capacity of elderly patients after hip fracture surgery.

As with previous studies [6] that assessed the functional parameters from continuous data, such as the Functional Independence Measure $[9,12]$ or cumulated ambulation score [13], we obtained similar results. Age, duration of admission, day from fracture to operation, day from operation to rehabilitation, functional status of gait before hip surgery, cognitive dysfunction, and postoperative complication were correlated with the short-term functional mobility after hip surgery. These results meant that functional grading by the use of ambulatory assistive devices could represent a patient's functional status to some extent.

In this sample of older patients who underwent hip fracture surgery, only 192 patients $(34.7 \%)$ achieved functional mobility before hip fracture at the point of one month after hip surgery. We found several factors that may help to predict the early recovery of pre-fracture mobility after a hip fracture. Advanced age and a male gender were known risk factors for mortality and functional deterioration after a hip fracture $[19,20]$. Although most former studies agreed that older age was an independent predictor of locomotion and mortality, the gender influence on functional outcomes following hip fracture re- mained controversial. Some authors have found no effect of gender on six months post-surgery ADL and walking ability [21]. In the present study, older people tended to have a less early recovery of functional mobility, but gender difference was not found to have a significant effect on either. In addition, older age had been identified as an independent risk factor for recurrent and injurious falls following a hip fracture [22]. Thus, we needed to consider the age factor in the rehabilitation of older patients after a hip fracture.

We found that the early recovery of functional mobility was associated with pre-injury cognitive function. The relationship between cognitive function and rehabilitation outcomes in elderly patients had been acknowledged in several studies [23-25]. Cognitively impaired patients showed a worse discharge functional level, and fewer were discharged to their own homes [25]. However, it should not be thought that cognitively impaired patients do not benefit from rehabilitation programs. In another study, cognitively impaired patients seemed to have the same functional gain as normal patients when the patients were SG in their pre-fracture state [24].

The result of the present study showed that patients who had depression showed less early recovery of functional mobility ( $16.7 \%$ vs. $35.1 \%$ ), but this result was not statistically significant. As a depression diagnosis was retrospectively determined based on each patient's medical history, it might be underestimated compared to patients' depressive symptoms. According to previous studies, depression negatively influenced rehabilitation outcomes and increased morbidity and mortality [26]. Because depression was a treatable condition, screening for depression in elderly patients with hip fracture and early management may improve patients' early recovery of functional mobility.

In some survival studies, patients with a trochanteric fracture showed a higher mortality risk than those with a neck fracture $[27,28]$. In the present study, it was also found that the early recovery of functional mobility was associated with the fracture type. Patients with trochanteric fracture showed a higher risk of deteriorating functional mobility than those with a neck fracture. However, unlike the other reports, we found no significant relationship between surgical methods and functional outcomes. According to previous studies, delaying the operation more than 48 hours was associated with increased com- 
plications and mortality rate [29]. In the present study, the mean surgical delay was 4.4 days, and $35 \%$ of patients underwent surgery within two days of their injury. Unlike other reports, we found no significant relationship between surgical timing and early functional recovery. However, we found that early rehabilitation from a hip operation was associated with the early recovery of functional mobility. Bed rest and the associated immobilization did not decrease the risk of postoperative surgical complications [30] but was associated with muscle weakness, fatigue, and postoperative complications, such as thromboembolism and pulmonary complications [31]. Duke at al. [32] reported that mobility on day two postsurgery was significant and a reliable predictor of independence in transfer and ambulation. Early aggressive rehabilitation should be encouraged after hip fracture surgery, if possible. Furthermore, further research into the specific training methods in the early postoperative phase was needed.

There was no significant difference in the early recovery of functional mobility between patients transferred to the department of rehabilitation medicine and others; we could not find the reason from the patient baseline characteristics. In patients transferred to the department of rehabilitation medicine, cognitive dysfunctions that adversely affected the recovery of functional mobility were significantly common.

There were some limitations in our study that should be considered when interpreting the results. First, the retrospective design had disadvantages regarding its limited control over how the available data were collected as data collection via chart review can often result in incomplete information. The functional status of 46 patients could not be evaluated because of their lack of medical records. Furthermore, we did not have a quantitative method for assessing cognitive function and depression. Specifically, the presence of depression and cognitive dysfunction was only recorded if it was listed in the past medical chart, admission record, or medication history. Only 35 patients had a Mini-Mental Status Examination in their medical records. Many hidden or relatively mild cognitive dysfunction and mood disorders could have been overlooked. Third, the severity of pain could not be evaluated because of the lack of medical records. Even though medication for pain control was prescribed after the surgery, pain severity varied, which could affect the recovery of functional mobility after a hip fracture. Finally, the primary outcome of this study, functional recovery over the short-term follow-up period was only assessed with a retrospective chart-review. A certain degree of misclassification cannot be completely ruled out.

Our analysis identified the age, cognitive function, underlying severe disease, and the delay between the operation and rehabilitation as risk factors associated with the early recovery of pre-injury functional mobility. Since most of these factors already existed at the time of admission, the correction of these factors by intervention by the medical team was unlikely. Therefore, additional focus was placed on the effects of early rehabilitation on the early functional recovery after hip fracture. We recommended that early rehabilitation should begin three days after the hip operation. Further prospective studies should be undertaken to define this correlation, with a focus on the presence of other confounding variables that may affect the rehabilitation potential.

\section{CONFLICT OF INTEREST}

No potential conflict of interest relevant to this article was reported.

\section{ACKNOWLEDGMENTS}

This research was supported by Basic Science Research Program through the National Research Foundation of Korea (NRF) funded by the Ministry of Education, Science and Technology (Grant No. 2013R1A1A1007404).

\section{REFERENCES}

1. Deandrea S, Bravi F, Turati F, Lucenteforte E, La Vecchia C, Negri E. Risk factors for falls in older people in nursing homes and hospitals: a systematic review and meta-analysis. Arch Gerontol Geriatr 2013;56:407-15.

2. Lim JY, Park WB, Oh MK, Kang EK, Paik NJ. Falls in a proportional region population in Korean elderly: incidence, consequences, and risk factors. J Korean Geriatr Soc 2010;14:8-17.

3. Burge R, Dawson-Hughes B, Solomon DH, Wong JB, King A, Tosteson A. Incidence and economic burden of osteoporosis-related fractures in the United States, 2005-2025. J Bone Miner Res 2007;22:465-75. 
4. Cooper C. The crippling consequences of fractures and their impact on quality of life. Am J Med 1997;103:12S-19S.

5. Dubljanin-Raspopovic E, Markovic Denic L, Marinkovic J, Grajic M, Tomanovic Vujadinovic S, Bumbasirevic $\mathrm{M}$. Use of early indicators in rehabilitation process to predict one-year mortality in elderly hip fracture patients. Hip Int 2012;22:661-7.

6. Kristensen MT. Factors affecting functional prognosis of patients with hip fracture. Eur J Phys Rehabil Med 2011;47:257-64.

7. Folden S, Tappen R. Factors influencing function and recovery following hip repair surgery. Orthop Nurs 2007;26:234-41.

8. Bellelli G, Noale M, Guerini F, Turco R, Maggi S, Crepaldi $\mathrm{G}$, et al. A prognostic model predicting recovery of walking independence of elderly patients after hipfracture surgery: an experiment in a rehabilitation unit in Northern Italy. Osteoporos Int 2012;23:2189200.

9. Semel J, Gray JM, Ahn HJ, Nasr H, Chen JJ. Predictors of outcome following hip fracture rehabilitation. PM R 2010;2:799-805.

10. Pugely AJ, Martin CT, Gao Y, Klocke NF, Callaghan JJ, Marsh JL. A risk calculator for short-term morbidity and mortality after hip fracture surgery. J Orthop Trauma 2014;28:63-9.

11. Savino E, Martini E, Lauretani F, Pioli G, Zagatti AM, Frondini $\mathrm{C}$, et al. Handgrip strength predicts persistent walking recovery after hip fracture surgery. Am J Med 2013;126:1068-75.e1.

12. Hershkovitz A, Kalandariov Z, Hermush V, Weiss R, Brill S. Factors affecting short-term rehabilitation outcomes of disabled elderly patients with proximal hip fracture. Arch Phys Med Rehabil 2007;88:916-21.

13. Foss NB, Kristensen MT, Kehlet H. Prediction of postoperative morbidity, mortality and rehabilitation in hip fracture patients: the cumulated ambulation score. Clin Rehabil 2006;20:701-8.

14. Faruqui SR, Jaeblon T. Ambulatory assistive devices in orthopaedics: uses and modifications. J Am Acad Orthop Surg 2010;18:41-50.

15. Mungas D. In-office mental status testing: a practical guide. Geriatrics 1991;46:54-8, 63, 66.

16. Svensson O, Stromberg L, Ohlen G, Lindgren U. Prediction of the outcome after hip fracture in elderly patients. J Bone Joint Surg Br 1996;78:115-8.

17. Bateni H, Maki BE. Assistive devices for balance and mobility: benefits, demands, and adverse consequences. Arch Phys Med Rehabil 2005;86:134-45.

18. IJzerman MJ, Baardman G, van 't Hof MA, Boom HB, Hermens HJ, Veltink PH. Validity and reproducibility of crutch force and heart rate measurements to assess energy expenditure of paraplegic gait. Arch Phys Med Rehabil 1999;80:1017-23.

19. Holt G, Smith R, Duncan K, Hutchison JD, Gregori A. Gender differences in epidemiology and outcome after hip fracture: evidence from the Scottish Hip Fracture Audit. J Bone Joint Surg Br 2008;90:480-3.

20. Lieberman D, Fried V, Castel H, Weitzmann S, Lowenthal MN, Galinsky D. Factors related to successful rehabilitation after hip fracture: a case-control study. Disabil Rehabil 1996;18:224-30.

21. Penrod JD, Litke A, Hawkes WG, Magaziner J, Doucette JT, Koval KJ, et al. The association of race, gender, and comorbidity with mortality and function after hip fracture. J Gerontol A Biol Sci Med Sci 2008;63:867-72.

22. Lloyd BD, Williamson DA, Singh NA, Hansen RD, Diamond TH, Finnegan TP, et al. Recurrent and injurious falls in the year following hip fracture: a prospective study of incidence and risk factors from the Sarcopenia and Hip Fracture study. J Gerontol A Biol Sci Med Sci 2009;64:599-609.

23. Morghen S, Gentile S, Ricci E, Guerini F, Bellelli G, Trabucchi M. Rehabilitation of older adults with hip fracture: cognitive function and walking abilities. J Am Geriatr Soc 2011;59:1497-502.

24. Beloosesky Y, Grinblat J, Epelboym B, Weiss A, Grosman B, Hendel D. Functional gain of hip fracture patients in different cognitive and functional groups. Clin Rehabil 2002;16:321-8.

25. Hagino T, Ochiai S, Sato E, Watanabe Y, Senga S, Haro H. Prognostic prediction in patients with hip fracture: risk factors predicting difficulties with discharge to own home. J Orthop Traumatol 2011;12:77-80.

26. Holmes J, House A. Psychiatric illness predicts poor outcome after surgery for hip fracture: a prospective cohort study. Psychol Med 2000;30:921-9.

27. Haentjens P, Autier P, Collins J, Velkeniers B, Vanderschueren D, Boonen S. Colles fracture, spine fracture, and subsequent risk of hip fracture in men and women: a meta-analysis. J Bone Joint Surg Am 2003;85- 
A:1936-43.

28. Lawton JO, Baker MR, Dickson RA. Femoral neck fractures: two populations. Lancet 1983;2:70-2.

29. Zhao Y, Fu D, Chen K, Li G, Cai Z, Shi Y, et al. Outcome of hemiarthroplasty and total hip replacement for active elderly patients with displaced femoral neck fractures: a meta-analysis of 8 randomized clinical trials. PLoS One 2014;9:e98071.

30. Kim JW, Byun SE, Chang JS. The clinical outcomes of early internal fixation for undisplaced femoral neck fractures and early full weight-bearing in elderly patients. Arch Orthop Trauma Surg 2014;134:941-6.

31. Upadhyay S, Taqi Raza HK. Four quadrant parallel peripheral screw fixation for displaced femoral neck fractures in elderly patients. Indian J Orthop 2014;48:226.

32. Duke RG, Keating JL. An investigation of factors predictive of independence in transfers and ambulation after hip fracture. Arch Phys Med Rehabil 2002;83:15864. 